Published in final edited form as:

Nat Rev Cardiol. 2017 September ; 14(9): 550-559. doi:10.1038/nrcardio.2017.70.

\title{
Physical function and exercise training in older patients with heart failure
}

\author{
Andrew J. Stewart Coats ${ }^{1,2}$, Daniel E. Forman ${ }^{3}$, Mark Haykowsky $^{4}$, Dalane W. Kitzman ${ }^{5}$, \\ Amy McNeil ${ }^{6}$, Tavis S. Campbell ${ }^{7}$, Ross Arena ${ }^{6}$ \\ ${ }^{1}$ Monash University, Clayton Campus, Melbourne, Victoria 3800, Australia. \\ 2University of Warwick, Kirby Corner Road, Coventry CV4 8UW, UK. \\ ${ }^{3}$ Section of Geriatric Cardiology, University of Pittsburgh Medical Center, VA Pittsburgh \\ Healthcare System, 259 Mt Nebo Pointe Drive, Pittsburgh, Pennsylvania 15213, USA. \\ ${ }^{4}$ College of Nursing and Health Innovation, University of Texas at Arlington, 411 South \\ Nedderman Drive, Arlington, Texas 76019, USA. \\ ${ }^{5}$ Cardiovascular Medicine Section, Department of Internal Medicine, Wake Forest School of \\ Medicine, 1 Medical Center Boulevard, Winston-Salem, North Carolina 27157, USA. \\ ${ }^{6}$ Department of Physical Therapy, Department of Kinesiology and Nutrition, College of Applied \\ Health Sciences, University of Illinois at Chicago, 1919 West Taylor Street, Chicago, Illinois \\ 60612, USA. \\ ${ }^{7}$ Department of Psychology, University of Calgary, 2500 University Drive North West, Calgary, \\ Alberta T2N 1N4, Canada.
}

\section{Abstract}

Heart failure (HF) is a common end point for numerous cardiovascular conditions, including coronary artery disease, valvular disease, and hypertension. HF predominantly affects older individuals (aged $\geq 70$ years), particularly those living in developed countries. The pathophysiological sequelae of HF progression have a substantial negative effect on physical function. Diminished physical function in older patients with HF, which is the result of combined disease-related and age-related effects, has important implications on health. A large body of research spanning several decades has demonstrated the safety and efficacy of regular physical activity in improving outcomes among the HF population, regardless of age, sex, or ethnicity. However, patients with HF, especially those who are older, are less likely to engage in regular exercise training compared with the general population. To improve initiation of regular exercise training and subsequent long-term compliance, there is a need to rethink the dialogue between

Correspondence to A.J.S.C. Monash University, Clayton Campus, Melbourne, Victoria 3800, Australia. ajscoats@aol.com. Author contributions

A.J.S.C. researched data and wrote the article. A.J.S.C. and D.E.F. contributed to discussion of content. All authors reviewed and edited the manuscript before submission.

Competing interests statement

The authors declare no competing interests.

Publisher's note

Springer Nature remains neutral with regard to jurisdictional claims in published maps and institutional affiliations. 
clinicians and patients. This Review discusses the need to improve physical function and exercise habits in patients with HF, focusing on the older population.

Heart failure (HF) is the common end point for numerous cardiovascular conditions, including coronary artery disease, valvular disease, and hypertension. Although HF can develop at any age, it predominantly affects those who are older (aged $\geq 70$ years) ${ }^{1}$. Older individuals with $\mathrm{HF}$ are more likely to be hospitalized more than once, resulting in substantial economic burden on the health-care system. As a consequence, a heightened focus has been placed on reducing hospital admissions associated with HF. Much of what is known about HF treatments that have been shown to reduce morbidity and mortality have largely been evaluated in younger cohorts of patients (aged < 70 years), and almost exclusively in those with HF with reduced ejection fraction (HFrEF). Older patients with HF often have a different clinical pattern with relatively preserved left ventricular (LV) systolic function, known as $\mathrm{HF}$ with preserved ejection fraction $(\mathrm{HFpEF})^{2}$. Although symptomatology and adverse outcomes associated with HF are similar between HFrEF and HFpEF, particularly with regard to reduced functional capacity, the evidence base for therapies in $\mathrm{HFpEF}$ is often lacking compared with $\mathrm{HFrEF}^{2}$. Therefore, the majority of research cited in this Review was conducted in patients with HFrEF, unless HFpEF is specifically mentioned.

The pathophysiological sequelae of HF have a substantial negative effect on all aspects of physical function. Diminished physical function, owing to decreased cardiorespiratory fitness and muscular strength in the older population with HF, is particularly concerning. These changes result in a reduced capacity to perform daily activities, and often underlie a very poor quality of life. The risk of falls is also increased in older patients with HF. Assessing the functional capacity of an older patient with HF is vital from multiple perspectives, such as risk prediction for future adverse events, assessment of disease severity, and guidance of medical management (including recommendations for physical activity and exercise training $)^{3}$.

Patients with stable HF should remain physically active and participate in a structured exercise programme. A substantial body of knowledge spanning several decades has clearly demonstrated the safety and benefits of regular physical activity and exercise training in the HF population ${ }^{4-6}$, regardless of age, sex, or ethnicity. These benefits including improvements in physiology, prognosis, physical function, and quality of life.

Unfortunately, physical activity patterns and engagement in regular exercise training are poor in the HF population, particularly in those who are aged $>70$ years ${ }^{7}$. In this Review, we address the need to improve the physical function and exercise habits in patients with HF, focusing on the older population. Improving communication between clinicians and patients, and increasing understanding of data examined under the health literacy framework, are important considerations in the search for strategies to improve the uptake of and adherence to physical activity. 


\section{Physiological effects of ageing and HF}

\section{Normal response to physical stimuli.}

The body's capacity to respond to a physical stimulus is dependent on the integration of several physiological systems, as well as the type and intensity of the stimulus. The cardiovascular, respiratory (musculature driving air exchange), pulmonary (ventilation-perfusion coupling), and peripheral skeletal muscles coordinate and integrate with neural, reflex, and hormonal control systems in response to a physical stimulus ${ }^{8}$. Physical stimuli can be divided into two broad categories: aerobic stimuli and muscle resistance stimuli. Response to an aerobic stimulus is dependent upon the capacity of the respiratory muscular system to augment air exchange, pulmonary system to augment gas exchange at the alveoli-pulmonary vasculature interface, cardiovascular system to augment cardiac output and delivery of oxygen to working skeletal muscle, and working skeletal muscle to augment oxygen consumption $\left(\mathrm{VO}_{2}\right)$ and increase aerobic energy production to meet the demand induced by a given level of exertion ${ }^{8}$. Although any of these systems, or combinations thereof, can be the rate-limiting step in the adequate response to an aerobic stimulus, insufficient augmentation of cardiac output is a common core culprit ${ }^{9}$. The response to a muscle resistance stimulus is dependent primarily on the capacity of working skeletal muscle to generate force for a single contraction (maximal force generation) or over several contractions (muscular endurance). This type of physical stimulus is exclusively or largely anaerobic in nature, and is dependent upon muscle cross-sectional area, neuronal firing pattern, and the contractile properties of muscle fibres.

\section{Physiological consequences of ageing.}

Normal ageing is associated with reduced physiological reserve capacity in a wide range of systems responsible for responses to aerobic and muscle resistance stimuli. The normal ageing process reduces cardiac function through several pathways, with a final common pathway being diminished diastolic function associated also with mild reductions in maximal systolic function ${ }^{10,11}$. An age-related decline in maximal heart rate has also been observed. The decline in maximal stroke volume and decrease in heart rate work synergistically to reduce cardiac output, which is the primary reason for the fall in peak $\mathrm{VO}_{2}$ in apparently healthy individuals ${ }^{12,13}$. The FRIEND registry ${ }^{12}$ reported that the 50th percentile peak $\mathrm{VO}_{2}$ declined from 48.0 to $24.4 \mathrm{ml} / \mathrm{kg} / \mathrm{min}$, and from 37.3 to $18.3 \mathrm{ml} / \mathrm{kg} / \mathrm{min}$ from the second decade to the seventh decade of life in apparently healthy men and women, respectively (see original publication for full decade-related declines across percentiles of peak $\mathrm{VO}_{2}$ ). Although these declines in cardiorespiratory fitness substantially reduce physical function across the lifespan, the slope of this decline can be attenuated by lifelong participation in appropriate levels of physical activity and structured exercise training. Ageing is also associated with changes in skeletal muscle characteristics, namely a decline in capacity for aerobic energy production, cross-sectional area, and fibre contractile properties $^{14}$. As such, age-related changes to skeletal muscle further contribute to the decline in capacity to respond to both an aerobic stimulus and a muscle resistance stimulus. Whereas other age-related changes to the respiratory, pulmonary, and cardiovascular systems exist, the changes associated with declined cardiac and skeletal muscle function are the primary drivers of reduced physical performance ${ }^{14}$. Notably, published normative tables and 
regression equations for age-specific and sex-specific peak $\mathrm{VO}_{2}$ provide an estimate that is influenced by many factors and, therefore, is subject to considerable prediction error. In particular, the data used to develop peak $\mathrm{VO}_{2}$ normative values and prediction equations have historically not included a large number of older patients. As such, care needs to be taken when extrapolating these values to older individuals, irrespective of health status (that is, from apparent health to confirmed chronic disease diagnosis).

\section{Physiological consequences of HF.}

As with ageing, a core pathophysiological issue associated with HF is diminished cardiac output. This substantial disease-related decline in cardiac output is attributable to systolic or diastolic dysfunction, as is seen in HFrEF or HFpEF, respectively. Systolic and diastolic dysfunction might also coexist, although the primary diagnosis is based on resting LV ejection fraction in the presence of a confirmed diagnosis of HF. Regardless of the mechanism of $\mathrm{HF}$, a considerable fall in cardiac output during physical exertion is a final common outcome that results in significantly lower peak $\mathrm{VO}_{2}$ levels compared with agepredicted and sex-predicted normal values ${ }^{15}$. Although this drop in cardiac output associated with $\mathrm{HF}$ is commonly thought to originate from the left ventricle, researchers are gaining an increased appreciation of the effect of HF on right-sided ventricular function and ventriculoarterial coupling in the presence of secondary pulmonary hypertension, a common occurrence in this population ${ }^{16}$. Moreover, the initial insult on cardiac function is accompanied by a near-simultaneous cascade of pathophysiological events that compromise multiple systems, all related to the capacity to perform activities that require physical exertion. Specifically, HF is associated with diminished respiratory muscle strength and endurance, pulmonary ventilation-perfusion matching, and skeletal muscle function. As such, HF commonly impairs the body's capacity to respond to both aerobic and muscle resistance stimuli, resulting in global impairment of physical function.

Established and long-standing HF is often characterized by a substantial decline in skeletal muscle mass associated with immune activation, neurohormonal overactivity, impaired anabolic hormone actions, and possibly cardiac cachexia ${ }^{17,18}$. In these patients, the accompanying changes in the peripheral musculature, peripheral vascular and endothelial function, and neurohormonal and cardiopulmonary reflex control systems might even overtake haemodynamic disturbance as the most important factor leading to impaired physical activity and functional capacity.

The changes in skeletal muscle function in older patients with HF have been shown to exaggerate reflex sympathoexcitation via muscle ergoreceptor overactivity, and this feature might worsen the severity of HF through its effects on persistent neurohormonal overactivity. Physical exercise training of the altered HF skeletal muscle has been shown to reduce this reflex sympathoexcitation at least partially ${ }^{19}$. Another feature of the elderly HF phenotype associated with exercise intolerance is impaired endothelial function; exercise training has been shown to correct this dysfunction more effec tively in older than in younger patients with $\mathrm{HF}$, through an increase in endothelial progenitor cells ${ }^{20}$.

Together, the age-related decline in physiological pathways associated with the capacity to respond to an exertional stimulus compounded by a HF diagnosis and its associated 
physiological derangements create a 'perfect storm' with respect to the potential for decline in physical function. These characteristics are summarized in TABLE 1. As such, older patients with HF should be considered to have a vulnerable phenotype requiring thorough assessment and aggressive interventional strategies when physical function is found to be compromised. The causes and the available strategies to address these problems and the effects they have on older individuals with HF are discussed below.

\section{Quantification of physical function}

The primary chronic symptom in older patients with either HFpEF or HFrEF, even in those who are clinically stable and nonoedematous, is severe exercise intolerance ${ }^{21}$. Exercise tolerance can be measured objectively during a cardiopulmonary exercise test as a decrease in peak $\mathrm{VO}_{2}$ (REF. 21). A study by Kitzman and co-workers found that, in older patients with $\mathrm{HF}$ (mean age $\geq 70$ years), peak $\mathrm{VO}_{2}$ during upright treadmill or cycling training was $\sim 30 \%$ lower than in age-matched healthy individuals, and the magnitude of the decline was similar in patients with $\mathrm{HFrEF}$ or $\mathrm{HFpEF}^{22}$. The peak $\mathrm{VO}_{2}$ in older patients with $\mathrm{HF}(\sim 14$ $\mathrm{ml} / \mathrm{kg} / \mathrm{min}$ ) was also well below the cut-off value $(18 \mathrm{ml} / \mathrm{kg} / \mathrm{min})$ often used to distinguish between high versus low physical function ${ }^{22-24}$. HF-mediated decline in peak $\mathrm{VO}_{2}$ is associated with decreased physical functional performance and has been consistently shown to be an independent predictor of poor outcomes ${ }^{25,26}$.

Physical function parameters provide a wealth of clinically valuable data and should be a cornerstone evaluation in most HF patient populations. A wealth of evidence is available demonstrating the value of assessing physical function in patients with HF, with a particular focus on assessment during activities that are aerobic in nature (for example, traditional or cardiopulmonary exercise testing or the 6-min walking test). The majority of the literature reporting on the utility of assessing physical function in HF, however, has focused on younger patient cohorts. Importantly, advanced age is not a contraindication for assessing physical function, and perhaps it is of even higher relevance and importance in older patients given the increased likelihood of compromised exercise function.

Cardiopulmonary exercise testing is the gold-standard assessment method for aerobic exercise capacity and should be performed whenever feasible. The methods for cardiopulmonary exercise testing in older patients are largely consistent with those used in younger patients, but tailored testing protocols and modes of exercise might be available for the older cohort. The protocol chosen to test improvement in maximal aerobic tolerance in an older patient should typically be conservative in nature (for example, the ramp protocol). In addition, testing using an ergometer rather than treadmill might be more appropriate in older patients who have problems with balance. Cardiopulmonary exercise testing can be highly reproducible in elderly patients with either HFrEF or $\mathrm{HFpEF}^{27,28}$. When cardiopulmonary exercise testing is not feasible (for example, owing to lack of equipment, appropriate expertise, or suitable level of supervision), other approaches to assessing aerobic performance should be employed, such as traditional exercise testing without ventilatory expired gas analysis or the 6-min walking test ${ }^{29}$. TABLE 2 provides a list of approaches to aerobic exercise testing and important considerations. 
In addition to aerobic exercise testing, assessment of physical function from a muscular strength and endurance perspective is of critical value in older patients, particularly with regard to assessing balance integrity, fall risk, and the ability to perform daily activities ${ }^{30}$. The assessment of muscular strength and endurance can be performed in several ways and is again dependent on available equipment, expertise, and appropriate levels of supervision ${ }^{31}$. Advanced physical function laboratories might have computerized isokinetic devices that can accurately quantify one-repetition maximum (maximum amount of force generated in one maximal contraction) and number of repetitions to fatigue. Noncomputerized standard resistance equipment can also be used. When resources are limited, use of field equipment (such as hand-held dynamometers) and tests (for example, timed up-and-go tests) are warranted. Finally, given the importance of respiratory muscle strength and endurance physical function in the HF population, the incorporation of this parameter into exercise testing is also strongly recommended. Hand-held computerized devices that allow for an accurate quantification of respiratory muscle strength and endurance are now readily available.

\section{Prognostic value of physical function.}

Cardiopulmonary exercise testing is the gold-standard measure of maximal aerobic power, and a number of physiological variables derived from cardiopulmonary testing (peak $\mathrm{VO}_{2}$ $<10 \mathrm{ml} / \mathrm{kg} / \mathrm{min}$, minute ventilation/carbon dioxide production slope $\geq 45$, presence of exercise oscillatory ventilation, and resting partial pressure of end-tidal carbon dioxide $<33$ $\mathrm{mmHg}$ and $<3 \mathrm{mmHg}$ increase during exercise) have been shown to have prognostic value in patients with $\mathrm{HF}^{32}$. Keteyian and co-workers examined the association between cardiopulmonary exercise testing-derived variables and all-cause mortality in 2,100 patients with HFrEF from the HF-ACTION trial ${ }^{25}$. In the multivariate analysis, adjusted for age and sex, the strongest predictors of all-cause mortality were peak $\mathrm{VO}_{2}$, percent-predicted peak $\mathrm{VO}_{2}$, and exercise duration ${ }^{25}$. Specifically, for each 5 percentage point-lowering of percentpredicted peak $\mathrm{VO}_{2}, 1 \mathrm{ml} / \mathrm{kg} / \mathrm{min}$-lowering of peak $\mathrm{VO}_{2}$, and 1-min-lowering of exercise duration, there was a $19 \%, 16 \%$, and $16 \%$ higher risk of all-cause mortality, respectively ${ }^{25}$. Shafiq and colleagues extended these findings to patients with HFpEF by demonstrating that peak $\mathrm{VO}_{2}$ and percent predicted peak $\mathrm{VO}_{2}$ were significantly associated with all-cause mortality or cardiac transplantation ${ }^{26}$. In particular, patients with $\mathrm{HFpEF}$ with a peak $\mathrm{VO}_{2}$ $\unlhd 3.9 \mathrm{ml} / \mathrm{kg} / \mathrm{min}$ and a percent-predicted peak $\mathrm{VO}_{2} \leq 58 \%$ had a cumulative 5 -year survival rate of $69 \%$ and $67 \%$, respectively. Taken together, these studies suggest that the marked impairment in peak $\mathrm{VO}_{2}$ and percent-predicted peak $\mathrm{VO}_{2}$ found in older patients with $\mathrm{HF}$ might be associated with a poor prognosis. Similarly, the MECKI Score study ${ }^{33}$ involving 3,794 patients with $\mathrm{HFrEF}$ reported that a score including peak $\mathrm{VO}_{2}$ and the slope of the relationship between minute ventilation and the rate of carbon dioxide production preserved its prognostic importance in older patients with their increased background mortality. A further study showed that the combination of impaired diastolic function and an abnormal exercise ventilatory response is predictive of a high-risk phenotype in the elderly patient with $\mathrm{HF}^{34}$.

Physical performance-based tests that are self-paced and simple to perform have also been shown to have important prognostic value in older patients with HF. For example, a 6-min 
walking distance $\ 300 \mathrm{~m}$ was associated with decreased survival in older (mean age $\geq 75$ years) patients with $\mathrm{HF}^{35,36}$. Moreover, Boxer and colleagues also reported that each $30 \mathrm{~m}$ reduction in 6-min walking distance was associated with a $19 \%$ increase in mortality in this patient cohort ${ }^{35}$. Impaired lower extremity physical function, as measured by the short physical performance battery (a series of physical tests measuring balance, gait, strength, and endurance by examining ability to stand with the feet together in the side-by-side, semitandem, and tandem positions, time to walk $8 \mathrm{ft}$, and time to rise from a chair and return to the seated position five times) at hospital discharge, has also been shown to be an independent predictor of long-term survival in older patients with HFrEF (mean age 80 years, range $65-101$ years $)^{37}$.

Older patients with cardiovascular disease, especially HF, are often frail and have unrecognized physical functional impairments in balance and mobility that are independent predictors of prognosis ${ }^{29}$. Importantly, these impairments should be assessed and targeted with individualized exercise prescriptions when initiating exercise interventions in order to prevent injuries ${ }^{38}$.

\section{Physical activity and exercise guidelines}

Numerous evidence-based sources are available for guidance on physical activity recommendations and for establishing an exercise regime in older patients with HF. Current physical activity guidelines state that all medically stable adults should accumulate $\geq 150$ min per week of moderate or $\geq 75 \mathrm{~min}$ per week of vigorous physical activity, or a combination thereof ${ }^{39}$. However, clinicians should recognize that these recommendations might seem like an insurmountable goal for many individuals, particularly those who have a sedentary lifestyle, but contemplate becoming more physically active. The issue of perceived ability to meet currently recommended physical activity goals is often more daunting for older individuals and is even further compounded by a diagnosis of HF. In addition, the current body of evidence, reflecting years of data and thousands of participants across the age, sex, and health spectrum, convincingly demonstrates the substantial improvement in health status and prognosis when an individual transitions from a sedentary lifestyle to increased physical activity that still falls far below current guideline recommendations ${ }^{40,41}$. For example, frail older patients who walked for $1 \mathrm{~h}$ per day had markedly lower mortality compared with those who did not ${ }^{42}$. Older patients with HF who are clinically stable should be prescribed with an individualized physical activity and exercise plan that the patient perceives to be achievable and is prepared to perform; aligns patient activity preferences and resources with physical activity and exercise recommendations; and aligns physical capabilities (such as good balance versus increased fall risk), real-time medical status (for example, physiologically stable versus exercise-induced arrhythmias), and level of supervision (that is, independent versus supervised). A physical activity and exercise plan should include both aerobic (for example, walking, cycling, or dancing) and muscular strength and endurance activities (for example, circuit weight training systems, elastic bands, or use of ones own body weight as resistance). Balance-focused activities, such as yoga or Tai Chi might also be advantageous, particularly for those who have problems with balance. In older patients with HF and respiratory muscle weak-ness, an inspiratory training programme should also be included. The combination of resistance and aerobic training has 
been shown to improve muscular strength and submaximal exercise tolerance in patients with HF, irrespective of age ${ }^{43}$. FIGURE 1 illustrates a decision tree for prescribing physical activity and exercise assessment, and counselling in the clinical setting. All contraindications to physical activity should be identified and screened, and exercise should be prescribed on a continual basis in patients with HF given the changing nature of the disease process (that is, compensated versus decompensated HF). A thorough review of contraindications is beyond the scope of this Review, and has been discussed previously ${ }^{44}$. When contraindications have been resolved, all patients should be prescribed an individually tailored physical activity and exercise plan.

\section{Improvement in exercise performance.}

Several investigators have examined the acute haemodynamic responses and chronic adaptations to endurance exercise training in patients with HFrEF aged $<65$ years ${ }^{45-47}$. Increased peak VO2 was found to be associated with a combination of favourable cardiac adaptations (increased maximal exercise cardiac output ${ }^{45-48}$ ), peripheral vascular changes (improved conduit artery endothelial function ${ }^{45,49}$, and decreased systemic and leg vascular resistance ${ }^{45,47}$ ), and skeletal muscle adaptations (increased oxidative muscle fibres ${ }^{50}$, elevated oxidative enzyme activity and capacity ${ }^{46}$, and reduced muscle wasting ${ }^{51}$ ) that resulted in increased muscle blood flow and extraction of oxygen by the active muscles ${ }^{46,52}$.

At present, there is a relative paucity of studies that have examined the central and peripheral adaptations to physical conditioning in patients with HF aged $>60$ years. Sandri and colleagues assessed the effects of 4 weeks of supervised cycle exercise training on peak $\mathrm{VO}_{2}$ and resting LV systolic and diastolic function in older ( $<65$ years, mean age $72 \pm 5$ years) patients with $\mathrm{HFrEF}^{53}$. The increased peak $\mathrm{VO}_{2}$ after training was associated with an improvement in LV ejection fraction, early (E) to late transmitral filling ratio, septal and lateral systolic and diastolic ( $\left.\mathrm{E}^{\prime}\right)$ myocardial annular tissue velocities and decreased mitral E-wave deceleration time, isovolumetric relaxation time, and septal and lateral E/E' (REF. $53)$.

Kitzman and co-workers have also shown that, in older patients with HFpEF, endurance exercise training does not seem to change resting LV systolic or diastolic function significantly ${ }^{54-57}$. These findings were extended in a further study demonstrating that the improvement in peak $\mathrm{VO}_{2}$ after 16 weeks of endurance exercise training in older patients with HFpEF was attributable to noncardiac peripheral factors, as measured by a significant increase in estimated peak arterial-venous oxygen difference with no significant overall change in cardiac output ${ }^{58}$. Two subsequent follow-up studies reported that continuous endurance exercise training or high-intensity interval training did not improve carotid artery distensibility, carotid-arterial pulse wave velocity, or brachial artery endothelial function in older patients with $\mathrm{HFpEF}^{54,56}$. Taken together, these data suggest that, in patients aged $>65$ years with $\mathrm{HFrEF}$, the increased peak $\mathrm{VO}_{2}$ after endurance training is a result of favourable skeletal muscle and/or microvascular adaptations that lead to increased oxygen transport and utilization by the active muscles. 


\section{Improvement in physical function.}

Numerous investigators have shown that endurance exercise training performed alone or with supplemental resistance training increases peak $\mathrm{VO}_{2}$ (REFS 53,55,58-60), 6-min walking distance ${ }^{55,60-62}$, and quality of life ${ }^{59,60,62}$ in the older HF population. The magnitude of the improvement in exercise capacity might be related to the intensity of exercise prescribed, as Wisloff and colleagues reported that high-intensity interval training (90-95\% peak heart rate for $4 \mathrm{~min}$, interspersed by $3 \mathrm{~min}$ of exercise performed at $50-70 \%$ peak heart rate) was superior to moderate-intensity continuous exercise ( $70-75 \%$ peak heart rate) for improvement in brachial artery endothelial function, vastus lateralis mitochondrial function, and peak $\mathrm{VO}_{2}$ in older (mean age 75 years) male patients with $\mathrm{HFrEF}^{59}$. However, the larger SMARTEX study ${ }^{63}$ found that high-intensity interval training and moderateintensity continuous training resulted in similar improvements in peak $\mathrm{VO}_{2}$ and $\mathrm{LV}$ remodelling in a group of patients with HFrEF. As such, the optimal aerobic training intensity for patients with HF has yet to be elucidated.

The improvement in functional outcomes might also be related to the mode of training performed. For example, high-intensity resistance training ( $80 \%$ one repetition maximum) significantly increased maximal muscular strength ${ }^{24,64}$, muscular endurance ${ }^{64}, 6$-min walking distance ${ }^{24,64}$, and physical functional performance ${ }^{24}$ without a change in peak $\mathrm{VO}_{2}$ in older patients with HFrEF.

Although relatively few studies assessing the influence of training protocol on HF outcomes have been performed to date, the available data suggest that the mechanisms underlying increased peak $\mathrm{VO}_{2}$ might depend partly on HF phenotype. In older patients with HFpEF, the increased peak $\mathrm{VO}_{2}$ after moderate-intensity continuous endurance exercise training was secondary to peripheral adaptations that result in increased peak exercise arterial-venous oxygen difference without a change in haemodynamic cardiovascular function (that is, no change in peak exercise cardiac output, central artery stiffness, or peripheral arterial endothelial function $)^{54,58}$. Furthermore, this improvement in peak $\mathrm{VO}_{2}$ has been associated with favourable adaptations in both cardiovascular and peripheral systems ${ }^{53,59}$. Improvements have also been described in LV diastolic function in both older and younger patients with HFrEF after 4 weeks of supervised endurance training ${ }^{61}$. In both HFpEF and $\mathrm{HFrEF}$, the peripheral contributions to improved peak $\mathrm{VO}_{2}$ with training seem to be largely attributable to improved skeletal muscle function (that is, increased percentage skeletal muscle oxidative fibres, mitochondrial volume density, oxidative capacity, capillarity, and muscle blood flow) ${ }^{46,52,65}$. This finding is not surprising given the skeletal muscle's capacity for rapid rejuvenation and remodelling, particularly compared with the myocardium ${ }^{21}$. Researchers have suggested that substantial evidence exists for improved peripheral oxygen utilization through both oxygen delivery and extraction via peripheral effects in patients with HFrEF or HFpEF ${ }^{66}$.

Together, these data indicate that older patients with either HFrEF or HFpEF have severely reduced physical function that is associated with reduced quality of life and poor outcomes that can be improved with exercise training. Future studies are required to determine whether exercise training-mediated improvements in physical function are associated with decreased hospitalization and improved survival in this patient cohort. Additional research is 
also needed to determine the optimal exercise intensity, the underlying mechanisms of exercise training improvement, and the interventions that can improve exercise adherence ${ }^{67}$. Furthermore, $>80 \%$ of older patients with HFpEF are overweight or obese, and multiple sources suggest that increased adipose tissue contributes to exercise intolerance in this setting 55,68,69. However, the role of caloric restriction in treatment of HF has been controversial owing to the 'HF paradox'; although increased body weight is a risk factor for the development of HF, after HF is established, increased body weight is associated with increased survival, possibly by the protective effect of improved energy stores ${ }^{70}$. Dietary weight loss via caloric restriction in older patients with HFpEF was shown to improve exercise capacity and quality of life, and these benefits were additive to aerobic exercise training ${ }^{55}$. Accordingly, future studies are required to examine exercise with other interventions (such as diet or medications) on health outcomes in older patients with HF. In this regard, data from a large observational study involving 4,623 patients with HFrEF suggest that the protective effect of obesity is lost if the patient has a low peak $\mathrm{VO}_{2}$, indicating that patients need to be at an optimal state before exercise training to achieve optimal physical fitness ${ }^{71}$.

\section{Approaches to improving health}

\section{Health literacy and communication.}

The importance of clinician-patient interaction and communication has gained increasing recognition in the past few years. Health literacy is defined as "the degree to which an individual has the capacity to obtain, communicate, process, and understand basic health information and services to make appropriate health decisions" (REF. 72). An estimated $12 \%$ of adults in the USA are thought to be proficient in health literacy; elderly individuals are at particularly high likelihood of having low health literacy ${ }^{73}$. A consensus statement from the Heart Failure Society of America recognized low health literacy as an important problem among the HF population ${ }^{74}$. Lower health literacy has been associated with poorer quality of life ${ }^{75}$, as well as an increased risk of adverse events ${ }^{74-76}$ in patients with chronic disease. Furthermore, low health literacy is related to lower self-care behaviour ${ }^{77}$, as well as lower physical function cross-sectionally and a faster health decline longitudinally ${ }^{78}$.

Health literacy, within its broader framework, includes physical literacy, defined as "the motivation, confidence, physical competence, knowledge, and understanding to maintain physical activity throughout the life course" (REF. 79). Elderly patients with HF are likely to lack most or all of the attributes of physical literacy. Given the potential to improve adherence with exercise interventions in elderly patients with HF, assessment of physical literacy should be a high priority in this population.

Although unintentional, health literacy can be misconstrued as unidirectional; a patient might be viewed as either literate or illiterate, which then affects adherence with health-care interventions and outcomes. In this setting, the patient is viewed as a passive recipient of information that the clinician provides. In 2017, McNeil and Arena proposed a concept termed Health Harmonics, defined as the two-way transaction of health information between a clinician and a patient that allows for an authentic, positive construction of meaning, rather than the passive deposition of information ${ }^{80}$. Future approaches to health literacy and 
communication should encourage patients to be their own advocate in the decision-making process and plan for improving their health, including adherence to an exercise programme.

\section{Use of behavioural coaching strategies.}

Despite strong evidence supporting the benefits of physical activity in patients with HF, including increased survival rates and decreased hospitalization rates, there is often poor uptake of prescribed exercise programmes, with many studies reporting nonadherence in the majority of patients ${ }^{81-84}$. Indeed, most HF hospital readmissions are associated with failed self-management behaviours, including nonadherence to an exercise regime ${ }^{85,86}$. These findings are not surprising, given that successful disease self-management in HF requires the adoption of several concurrent, challenging, and often novel health behaviour changes in addition to physical activity and exercise, including changes to diet and fluid intake, weight loss, complex medication regimen, and frequent medical appointments. In order to promote chronic disease self-management, health-care providers have traditionally employed a communication style that involves the unsolicited provision of expert opinion and directive advice. Whereas knowledge of the benefits of physical activity and exercise and prescription of lifestyle changes might be necessary, these factors are generally found to be insufficient to motivate long-term health behaviour change ${ }^{87}$. Motivational communication is an alternative set of evidence-based and patient-centred strategies and interventions that have been demonstrated to improve adherence to a wide range of health behaviours ${ }^{88-90}$. These techniques might include a combination of cognitive-behavioural strategies ${ }^{91,92}$, motivational interviewing, and interventions based on well-established theories of motivation (for example, self-determination theory ${ }^{93}$, social cognitive theory ${ }^{94}$, theory of planned behaviour ${ }^{95}$, and transtheoretical model ${ }^{96}$ ). A defining feature of motivational communication is the use of a counselling style based on motivational interviewing that respects patient autonomy and decisions about health behaviours, while attempting to resolve ambivalence around behaviour change by eliciting personal reasons for the change. A rapidly expanding body of evidence demonstrating the efficacy of various motivational communication approaches across a wide variety of health-care settings support increased physical activity duration and/or intensity, reduced activity limitation, and increased exercise self-efficacy (an important predictor of the adoption and maintenance of exercise behaviour) for improved health outcomes ${ }^{90,97}$.

Although nonadherence is often viewed as a patient-related issue, it is actually a multidimensional problem that also includes system-related and provider-related factors. Whereas motivational communication strategies that promote uptake of positive physical activity and exercise behaviours are recommended in ACC/AHA clinical practice guidelines for the management of $\mathrm{HF}^{98}$, the type of skills and preparation required to develop competency in delivery of these motivational strategies are unclear. Preliminary training recommendations, including considerations on duration, ongoing individualized supervision, and clinical decision-making have been reported elsewhere ${ }^{99}$. 


\section{Conclusions}

Physical activity and exercise training are important for the prevention of disease across the health spectrum. A wealth of evidence supports the adoption of an exercise programme in younger patients with HF. Although there is clear scientific justification for exercise training in the older HF population given its benefits on physiological, functional, and clinical outcomes, there seems to be a low rate of adoption of exercise training in older patients with HF. All stable patients with HF, irrespective of age, should be encouraged to engage routinely in physical activity and a structured exercise training programme. Novel strategies are available with the aim of improving adherence to prescribed exercise regimens in patients.

\section{References}

1. Ponikowski P. et al. 2016 ESC guidelines for the diagnosis and treatment of acute and chronic heart failure: the task force for the diagnosis and treatment of acute and chronic heart failure of the European Society of Cardiology (ESC). Developed with the special contribution of the Heart Failure Association (HFA) of the ESC. Eur. J. Heart Fail 18, 891-975 (2016). [PubMed: 27207191]

2. Coats AJ \& Shewan L. The management of heart failure with preserved ejection fraction (HFpEF). Int. Cardiovasc. Forum J 1, 108-112 (2014).

3. Forman DE et al. Prioritizing functional capacity as a principal end-point for therapies oriented to older adults with cardiovascular disease. A scientific statement for healthcare professionals from the American Heart Association. Circulation 135, e894-e918 (2017). [PubMed: 28336790]

4. Coats AJ Heart failure: support for exercise training in CHF. Nat. Rev. Cardiol 6, 447-448 (2009). [PubMed: 19554002]

5. Dieberg G, Ismail H, Giallauria F. \& Smart NA Clinical outcomes and cardiovascular responses to exercise training in heart failure patients with preserved ejection fraction: a systematic review and meta-analysis. J. Appl. Physiol. (1985) 119, 726-733 (2015). [PubMed: 25749444]

6. Hsu CY, Hsieh PL, Hsiao SF \& Chien MY Effects of exercise training on autonomic function in chronic heart failure: systematic review. Biomed Res. Int 2015, 591708 (2015).

7. Pozehl B, McGuire R. \& Norman J. Team-based care for cardiac rehabilitation and exercise training in heart failure. Heart Fail. Clin 11, 431-449 (2015). [PubMed: 26142640]

8. Xiang L. \& Hester RL Cardiovascular responses to exercise Colloquium series on integrated systems physiology: from molecule to function. Vol.3 No.7 1-124 (Morgan \& Claypool Life Sciences, 2011).

9. Wilson JR, Rayos G, Yeoh TK, Gothard P. \& Bak K. Dissociation between exertional symptoms and circulatory function in patients with heart failure. Circulation 92, 47-53 (1995). [PubMed: 7788915]

10. Xu B. \& Daimon M. Cardiac aging phenomenon and its clinical features by echocardiography. J. Echocardiogr 14, 139-145 (2016). [PubMed: 27169598]

11. Vancheri F, Vancheri S. \& Henein MY Effect of age on left ventricular global dyssynchrony in asymptomatic individuals: a population study. Echocardiography 33, 977-983 (2016). [PubMed: 27018994]

12. Kaminsky LA, Arena R. \& Myers J. Reference standards for cardiorespiratory fitness measured with cardiopulmonary exercise testing: data from the Fitness Registry and the Importance of Exercise National Database. Mayo Clin. Proc 90, 1515-1523 (2015). [PubMed: 26455884]

13. Kaminsky LA et al. The importance of cardiorespiratory fitness in the United States: the need for a national registry: a policy statement from the American Heart Association. Circulation 127, 652662 (2013). [PubMed: 23295916]

14. Goodpaster BH et al. The loss of skeletal muscle strength, mass, and quality in older adults: the Health, Aging and Body Composition Study. J. Gerontol. A Biol. Sci. Med. Sci 61, 1059-1064 (2006). [PubMed: 17077199] 
15. Williams SG et al. Peak exercise cardiac power output; a direct indicator of cardiac function strongly predictive of prognosis in chronic heart failure. Eur. Heart J 22, 1496-1503 (2001). [PubMed: 11482923]

16. Moraes DL, Colucci WS \& Givertz MM Secondary pulmonary hypertension in chronic heart failure: the role of the endothelium in pathophysiology and management. Circulation 102, 17181723 (2000). [PubMed: 11015353]

17. Josiak K, Jankowska EA, Piepoli MF, Banasiak W. \& Ponikowski P. Skeletal myopathy in patients with chronic heart failure: significance of anabolic-androgenic hormones. J. Cachexia Sarcopenia Muscle 5, 287-296 (2014). [PubMed: 25081949]

18. Sente T, Van Berendoncks AM, Hoymans VY \& Vrints CJ Adiponectin resistance in skeletal muscle: pathophysiological implications in chronic heart failure. J. Cachexia Sarcopenia Muscle 7 , 261-274 (2016). [PubMed: 27239409]

19. Nobre TS et al. Post-exercise neurovascular control in chronic heart failure patients. Int. J. Sports Med 37, 1073-1079 (2016). [PubMed: 27676142]

20. Sandri M. et al. Chronic heart failure and aging - effects of exercise training on endothelial function and mechanisms of endothelial regeneration: results from the Leipzig Exercise Intervention in Chronic heart failure and Aging (LEICA) study. Eur. J. Prev. Cardiol 23, 349-358 (2016). [PubMed: 26015451]

21. Haykowsky MJ, Tomczak CR, Scott JM, Paterson DI \& Kitzman DW Determinants of exercise intolerance in patients with heart failure and reduced or preserved ejection fraction. J. Appl. Physiol. (1985) 119, 739-744 (2015). [PubMed: 25911681]

22. Kitzman DW et al. Pathophysiological characterization of isolated diastolic heart failure in comparison to systolic heart failure. JAMA 288, 2144-2150 (2002). [PubMed: 12413374]

23. Bhella PS et al. Abnormal haemodynamic response to exercise in heart failure with preserved ejection fraction. Eur. J. Heart Fail 13, 1296-1304 (2011). [PubMed: 21979991]

24. Savage PA et al. Effect of resistance training on physical disability in chronic heart failure. Med. Sci. Sports Exerc 43, 1379-1386 (2011). [PubMed: 21233772]

25. Keteyian SJ et al. Variables measured during cardiopulmonary exercise testing as predictors of mortality in chronic systolic heart failure. J. Am. Coll. Cardiol 67, 780-789 (2016). [PubMed: 26892413]

26. Shafiq A. et al. Prognostic value of cardiopulmonary exercise testing in heart failure with preserved ejection fraction. The Henry Ford HospITal CardioPulmonary EXercise Testing (FIT-CPX) project. Am. Heart J 174, 167-172 (2016). [PubMed: 26995385]

27. Marburger CT, Brubaker PH, Pollock WE, Morgan TM \& Kitzman DW Reproducibility of cardiopulmonary exercise testing in elderly patients with congestive heart failure. Am. J. Cardiol 82, 905-909 (1998). [PubMed: 9781977]

28. Scott JM et al. Reliability of peak exercise testing in patients with heart failure with preserved ejection fraction. Am. J. Cardiol 110, 1809-1813 (2012). [PubMed: 22981266]

29. Olsson LG, Swedberg K, Clark AL, Witte KK \& Cleland JG Six minute corridor walk test as an outcome measure for the assessment of treatment in randomized, blinded intervention trials of chronic heart failure: a systematic review. Eur. Heart J 26, 778-793 (2005). [PubMed: 15774495]

30. Izawa KP et al. The relation between Geriatric Nutritional Risk Index and muscle mass, muscle strength, and exercise capacity in chronic heart failure patients. Int. J. Cardiol 177, 1140-1141 (2014). [PubMed: 25156851]

31. Cuthbert SC \& Goodheart GJ Jr. On the reliability and validity of manual muscle testing: a literature review. Chiropr. Osteopat 15, 4 (2007). [PubMed: 17341308]

32. Guazzi M. et al. EACPR/AHA scientific statement. Clinical recommendations for cardiopulmonary exercise testing data assessment in specific patient populations. Circulation 126, 2261-2274 (2012). [PubMed: 22952317]

33. Carubelli V. et al. Exercise performance is a prognostic indicator in elderly patients with chronic heart failure - application of metabolic exercise cardiac kidney indexes score. Circ. J 79, 26082615 (2015). [PubMed: 26477272] 
34. Malfatto G. et al. Diastolic dysfunction and abnormal exercise ventilation predict adverse outcome in elderly patients with chronic systolic heart failure. Eur. J. Prev. Cardiol 19, 396-403 (2012). [PubMed: 21450576]

35. Boxer R. et al. The 6-minute walk is associated with frailty and predicts mortality in older adults with heart failure. Congest. Heart Fail 16, 208-213 (2010). [PubMed: 20887617]

36. Chamberlain AM et al. Physical health status measures predict all-cause mortality in patients with heart failure. Circ. Heart Fail 6, 669-675 (2013). [PubMed: 23625946]

37. Chiarantini D. et al. Lower extremity performance measures predict long-term prognosis in older patients hospitalized for heart failure. J. Card. Fail 16, 390-395 (2010). [PubMed: 20447574]

38. Reeves GR et al. Comparison of frequency of frailty and severely impaired physical function in patients $\searrow 60$ years hospitalized with acute decompensated heart failure versus chronic stable heart failure with reduced and preserved left ventricular ejection fraction. Am. J. Cardiol 117, 19531958 (2016). [PubMed: 27156830]

39. World Health Organization. Physical activity and adults. Global Strategy on Diet, Physical Activity and Health http://www.who.int/dietphysicalactivity/factsheet_adults/en/ (2017).

40. Warburton DE, Nicol CW \& Bredin SS Health benefits of physical activity: the evidence. CMAJ 174, 801-809 (2006). [PubMed: 16534088]

41. Pate RR et al. Physical activity and public health: a recommendation from the Centers for Disease Control and Prevention and the American College of Sports Medicine. JAMA 273, 402-407 (1995). [PubMed: 7823386]

42. Landi F. et al. Walking one hour or more per day prevented mortality among older persons: results from ilSIRENTE study. Prev. Fail 47, 422-426 (2008).

43. Swank AM, Funk DC, Manire JT, Allard AL \& Denny DM Effect of resistance training and aerobic conditioning on muscular strength and submaximal fitness for individuals with chronic heart failure: influence of age and gender. J. Strength Cond. Res 24, 1298-1305 (2010). [PubMed: 20386473]

44. Fletcher GF et al. Exercise standards for testing and training: a scientific statement from the American Heart Association. Circulation 128, 873-934 (2013). [PubMed: 23877260]

45. Hambrecht R. et al. Effects of exercise training on left ventricular function and peripheral resistance in patients with chronic heart failure: a randomized trial. JAMA 283, 3095-3101 (2000). [PubMed: 10865304]

46. Hambrecht R. et al. Physical training in patients with stable chronic heart failure: effects on cardiorespiratory fitness and ultrastructural abnormalities of leg muscles. J. Am. Coll. Cardiol 25, 1239-1249 (1995). [PubMed: 7722116]

47. Coats AJ et al. Controlled trial of physical training in chronic heart failure. Exercise performance, hemodynamics, ventilation, and autonomic function. Circulation 85, 2119-2131 (1992). [PubMed: 1591831]

48. Dubach P. et al. Effect of high intensity exercise training on central hemodynamic responses to exercise in men with reduced left ventricular function. J. Am. Coll. Cardiol 29, 1591-1598 (1997). [PubMed: 9180124]

49. Linke A. et al. Endothelial dysfunction in patients with chronic heart failure: systemic effects of lower-limb exercise training. J. Am. Coll. Cardiol 37, 392-397 (2001). [PubMed: 11216952]

50. Hambrecht R. et al. Effects of endurance training on mitochondrial ultrastructure and fiber type distribution in skeletal muscle of patients with stable chronic heart failure. J. Am. Coll. Cardiol 29, 1067-1073 (1997). [PubMed: 9120161]

51. Gielen S. et al. Exercise training attenuates MuRF-1 expression in the skeletal muscle of patients with chronic heart failure independent of age: the randomized Leipzig Exercise Intervention in Chronic Heart Failure and Aging catabolism study. Circulation 125, 2716-2727 (2012). [PubMed: 22565934]

52. Sullivan MJ, Higginbotham MB \& Cobb FR Exercise training in patients with severe left ventricular dysfunction. Hemodynamic and metabolic effects. Circulation 78, 506-515 (1988). [PubMed: 3409495] 
53. Sandri M. et al. Age-related effects of exercise training on diastolic function in heart failure with reduced ejection fraction: the Leipzig Exercise Intervention in Chronic Heart Failure and Aging (LEICA) Diastolic Dysfunction Study. Eur. Heart J 33, 1758-1768 (2012). [PubMed: 22267243]

54. Kitzman DW et al. Effect of endurance exercise training on endothelial function and arterial stiffness in older patients with heart failure and preserved ejection fraction: a randomized, controlled, single-blind trial. J. Am. Coll. Cardiol 62, 584-592 (2013). [PubMed: 23665370]

55. Kitzman DW et al. Effect of caloric restriction or aerobic exercise training on peak oxygen consumption and quality of life in obese older patients with heart failure with preserved ejection fraction: a randomized clinical trial. JAMA 315, 36-46 (2016). [PubMed: 26746456]

56. Angadi SS et al. High-intensity interval training versus moderate-intensity continuous exercise training in heart failure with preserved ejection fraction: a pilot study. J. Appl. Physiol. (1985) 119, 753-758 (2015). [PubMed: 25190739]

57. Smart NA, Haluska B, Jeffriess L. \& Leung D. Exercise training in heart failure with preserved systolic function: a randomized controlled trial of the effects on cardiac function and functional capacity. Congest. Heart Fail 18, 295-301 (2012). [PubMed: 22536983]

58. Haykowsky MJ et al. Effect of endurance training on the determinants of peak exercise oxygen consumption in elderly patients with stable compensated heart failure and preserved ejection fraction. J. Am. Coll. Cardiol 60, 120-128 (2012). [PubMed: 22766338]

59. Wisloff U. et al. Superior cardiovascular effect of aerobic interval training versus moderate continuous training in heart failure patients: a randomized study. Circulation 115, 3086-3094 (2007). [PubMed: 17548726]

60. Cider A, Schaufelberger M, Sunnerhagen KS \& Andersson B. Hydrotherapy - a new approach to improve function in the older patient with chronic heart failure. Eur. J. Heart Fail 5, 527-535 (2003). [PubMed: 12921815]

61. Owen A. \& Croucher L. Effect of an exercise programme for elderly patients with heart failure. Eur. J. Heart Fail 2, 65-70 (2000). [PubMed: 10742705]

62. Austin J, Williams R, Ross L, Moseley L. \& Hutchison S. Randomised controlled trial of cardiac rehabilitation in elderly patients with heart failure. Eur. J. Heart Fail 7, 411-417 (2005). [PubMed: 15718182]

63. Ellingsen O. et al. High intensity interval training in heart failure patients with reduced ejection fraction. Circulation 135, 839-849 (2017). [PubMed: 28082387]

64. Pu CT et al. Randomized trial of progressive resistance training to counteract the myopathy of chronic heart failure. J. Appl. Physiol. (1985) 90, 2341-2350 (2001). [PubMed: 11356801]

65. Esposito F, Reese V, Shabetai R, Wagner PD \& Richardson RS Isolated quadriceps training increases maximal exercise capacity in chronic heart failure: the role of skeletal muscle convective and diffusive oxygen transport. J. Am. Coll. Cardiol 58, 1353-1362 (2011). [PubMed: 21920265]

66. Hirai DM, Musch TI \& Poole DC Exercise training in chronic heart failure: improving skeletal muscle O2 transport and utilization. Am. J. Physiol. Heart Circ. Physiol 309, H1419-H1439 (2015). [PubMed: 26320036]

67. Fleg JL et al. Exercise training as therapy for heart failure: current status and future directions. Circ. Heart Fail 8, 209-220 (2015). [PubMed: 25605639]

68. Haykowsky MJ et al. Impaired aerobic capacity and physical functional performance in older heart failure patients with preserved ejection fraction: role of lean body mass. J. Gerontol. A Biol. Sci. Med. Sci 68, 968-975 (2013). [PubMed: 23525477]

69. Haykowsky MJ et al. Skeletal muscle composition and its relation to exercise intolerance in older patients with heart failure and preserved ejection fraction. Am. J. Cardiol 113, 1211-1216 (2014). [PubMed: 24507172]

70. Zafrir B. et al. Body surface area as a prognostic marker in chronic heart failure patients: results from the Heart Failure Registry of the Heart Failure Association of the European Society of Cardiology. Eur. J. Heart Fail 18, 859-868 (2016). [PubMed: 27198159]

71. Piepoli MF et al. Exercise tolerance can explain the obesity paradox in patients with systolic heart failure: data from the MECKI Score Research Group. Eur. J. Heart Fail 18, 545-553 (2016). [PubMed: 27135769] 
72. Centers for Disease Control. What is health literacy? CDC https://www.cdc.gov/healthliteracy/ learn/ (updated 13 Dec 2017).

73. Friel CJ Improving health outcomes for low health literacy heart failure patients. Home Healthc. Now 34, 434-439 (2016). [PubMed: 27580282]

74. McNaughton CD et al. Health literacy and mortality: a cohort study of patients hospitalized for acute heart failure. J. Am. Heart Assoc 4, e001799 (2015).

75. Omachi TA, Sarkar U, Yelin EH, Blanc PD \& Katz PP Lower health literacy is associated with poorer health status and outcomes in chronic obstructive pulmonary disease. J. Gen. Intern. Med 28, 74-81 (2013). [PubMed: 22890622]

76. Rubin DJ, Donnell-Jackson K, Jhingan R, Golden SH \& Paranjape A. Early readmission among patients with diabetes: a qualitative assessment of contributing factors. J. Diabetes Complicat 28, 869-873 (2014). [PubMed: 25087192]

77. Matsuoka S. et al. Health literacy is independently associated with self-care behavior in patients with heart failure. Patient Educ. Couns 99, 1026-1032 (2016). [PubMed: 26830514]

78. Smith SG et al. Low health literacy predicts decline in physical function among older adults: findings from the LitCog cohort study. J. Epidemiol. Community Health 69, 474-480 (2015). [PubMed: 25573701]

79. Whitehead M. Physical Literacy: Throughout the Lifecourse (Routledge, 2010).

80. McNeil A. \& Arena R. The evolution of health literacy and communication: introducing health harmonics. Prog. Cardiovasc. Dis 59, 463-470 (2017). [PubMed: 28216109]

81. Buck HG "Determinants of self-care behaviors in community-dwelling patients with heart failure". J. Cardiovasc. Nurs 24, 425 (2009). [PubMed: 19858950]

82. van der Wal MH et al. Compliance in heart failure patients: the importance of knowledge and beliefs. Eur. Heart J 27, 434-440 (2006). [PubMed: 16230302]

83. Dickson VV \& Riegel B. Are we teaching what patients need to know? Building skills in heart failure self-care. Heart Lung 38, 253-261 (2009). [PubMed: 19486796]

84. Evangelista LS \& Shinnick MA What do we know about adherence and self-care? J. Cardiovasc. Nurs 23, 250-257 (2008). [PubMed: 18437067]

85. Moser DK \& Watkins JF Conceptualizing self-care in heart failure: a life course model of patient characteristics. J. Cardiovasc. Nurs 23, 205-218 (2008). [PubMed: 18437061]

86. Riegel B, Vaughan Dickson V, Goldberg LR \& Deatrick JA Factors associated with the development of expertise in heart failure self-care. Nurs. Res 56, 235-243 (2007). [PubMed: 17625462]

87. Lawlor DA \& Hanratty B. The effect of physical activity advice given in routine primary care consultations: a systematic review. J. Public Health Med 23, 219-226 (2001). [PubMed: 11585195]

88. Rubak S, Sandbaek A, Lauritzen T. \& Christensen B. Motivational interviewing: a systematic review and meta-analysis. Br. J. Gen. Pract 55, 305-312 (2005). [PubMed: 15826439]

89. Lundahl BW, Kunz C, Brownell C, Tollefson D. \& Burke BLA Meta-analysis of motivational interviewing: twenty-five years of empirical studies. Res. Soc. Work Pract 20, 137-160 (2010).

90. McGrane N, Galvin R, Cusack T. \& Stokes E. Addition of motivational interventions to exercise and traditional physiotherapy: a review and meta-analysis. Physiotherapy 101, 1-12 (2015). [PubMed: 25239472]

91. Beck JS Cognitive Behavior Therapy: Basics and Beyond (Guilford Press, 2011).

92. Artinian NT et al. Interventions to promote physical activity and dietary lifestyle changes for cardiovascular risk factor reduction in adults: a scientific statement from the American Heart Association. Circulation 122, 406-441 (2010). [PubMed: 20625115]

93. Deci EL \& Ryan RM Intrinsic Motivation and Self-Determination in Human Behavior (Plenum, 1985).

94. Bandura A. Self-efficacy: toward a unifying theory of behavioral change. Psychol. Rev 84, 191215 (1977). [PubMed: 847061]

95. Ajzen I. The theory of planned behavior. Organ. Behav. Hum. Decis. Process 50, 179-211 (1991). 
96. Prochaska JO \& Velicer WF The transtheoretical model of health behavior change. Am. J. Health Promot 12, 38-48 (1997). [PubMed: 10170434]

97. O'Halloran PD et al. Motivational interviewing to increase physical activity in people with chronic health conditions: a systematic review and meta-analysis. Clin. Rehabil 28, 1159-1171 (2014). [PubMed: 24942478]

98. Hunt SA et al. 2009 focused update incorporated into the ACC/AHA 2005 guidelines for the diagnosis and management of heart failure in adults: a report of the American College of Cardiology Foundation/ American Heart Association task force on practice guidelines: developed in collaboration with the International Society for Heart and Lung Transplantation. Circulation 119, e391-e479 (2009). [PubMed: 19324966]

99. Rouleau CR et al. Training healthcare providers in motivational communication for promoting physical activity and exercise in cardiometabolic health settings: do we know what we are doing? Curr. Cardiovasc. Risk Rep 9, 29 (2015). 


\section{Key points}

- $\quad$ Older patients (aged $\geq 70$ years) with heart failure (HF) have substantially impaired physical function and poor clinical outcomes

- A comprehensive assessment of physical function is strongly recommended in this cohort, and should ideally include assessment of aerobic performance as well as peripheral and respiratory muscle strength and endurance

- $\quad$ Physical activity and exercise training are important for improving outcomes in patients with HF, especially elderly patients, but uptake remains poor

- To improve long-term compliance with exercise training, there is a need to improve two-way communication between clinicians and patients, including comprehension of health information such as exercise training

- Improving health literacy, defined as 'the motivation, confidence, physical competence, knowledge, and understanding to maintain physical activity throughout the life course', is essential for improving exercise adherence in elderly patients with $\mathrm{HF}$ 


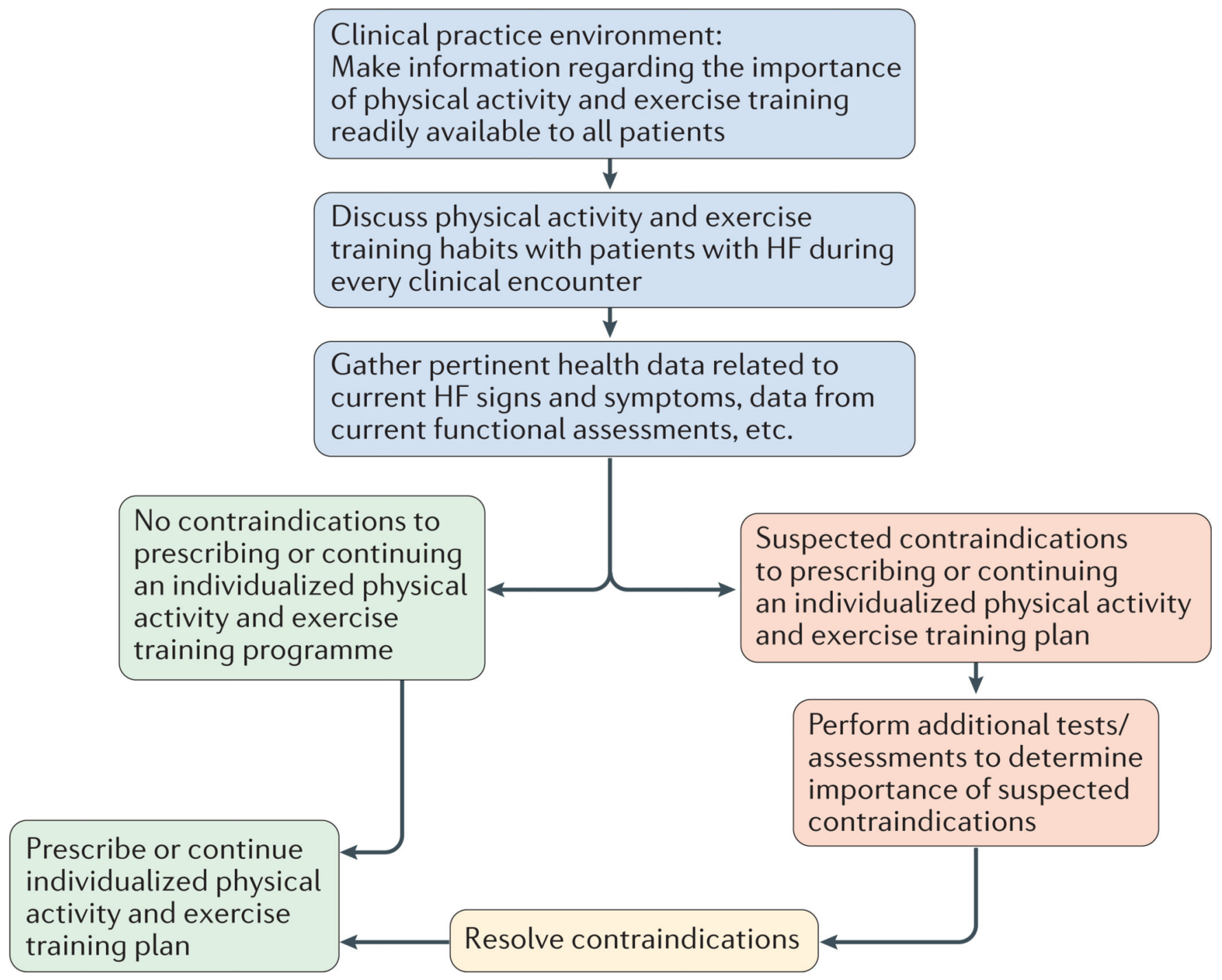

Figure 1|. Decision tree integrating the assessment, discussion, and prescription of physical activity and exercise in clinical practice.

This figure outlines the decision-making steps when advising an elderly patient with heart failure on an exercise training regimen. The figure includes a checklist of relative contraindications to exercise and an approach to how these could be managed or addressed. $\mathrm{HF}$, heart failure. 


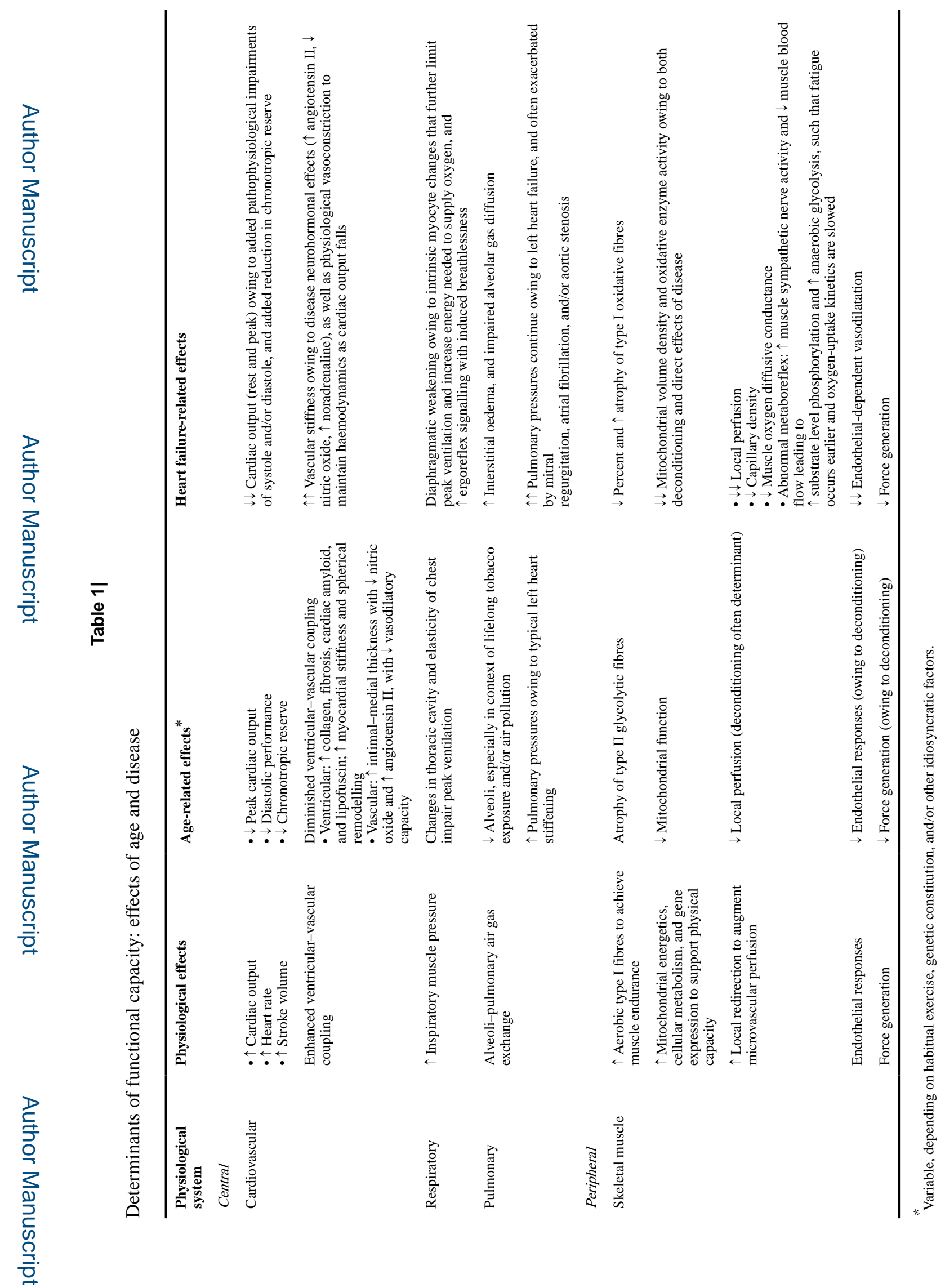

Nat Rev Cardiol. Author manuscript; available in PMC 2020 May 24. 


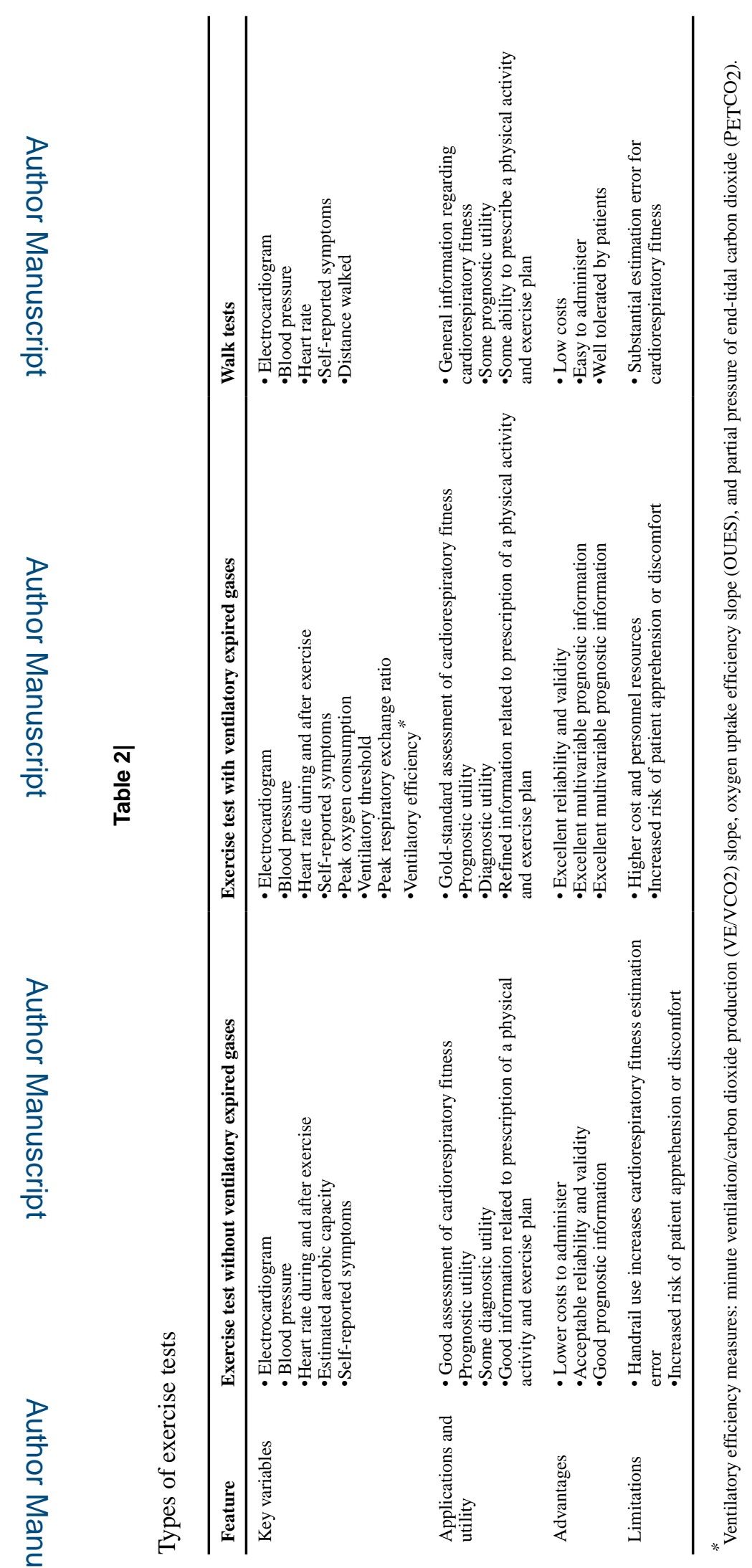

